\title{
The Effects of Systemic Therapy of PEGylated NEL-Like Protein 1 (NELL-1) on Fracture Healing in Mice
}

Justine Tanjaya, ${ }^{*}$ Elizabeth L. Lord, ${ }^{\dagger}$ Chenchao Wang, ${ }^{\ddagger \S}$ Yulong Zhang, ${ }^{\mathbb{f} \|}$ Jong K. Kim, * Alan Nguyen, * Llyod Baik, * Hsin C. Pan, ${ }^{*}$ Eric Chen, ${ }^{*}$ Jin H. Kwak, ${ }^{*}$ Xinli Zhang, ${ }^{*}$ Benjamin Wu, ${ }^{\top \|}$ Chia Soo, ${ }^{\dagger \ddagger}$ and Kang Ting ${ }^{*}$

From the Division of Growth and Development and the Section of Orthodontics, * School of Dentistry, the Department of Orthopaedic Surgery and the Orthopaedic Hospital Research Center ${ }^{\dagger}$ the Division of Plastic and Reconstructive Surgery, ${ }^{\ddagger}$ Department of Surgery, David Geffen School of Medicine, and the Departments of Bioengineering "and Materials Science and Engineering," University of California, Los Angeles, Los Angeles, California; and the Department of Plastic Surgery, ${ }^{\S}$ First Hospital of China Medical University, Shenyang, China

Accepted for publication November 21, 2017.

Address correspondence to Kang Ting, D.M.D, D.Med.Sc., School of Dentistry, University of California, Los Angeles, CHS 30-117, 10833 Le Conte Ave, Los Angeles, CA 90095. E-mail: kting@ dentistry.ucla.edu.

\begin{abstract}
Fractures are common, with an incidence of 13.7 per 1000 adults annually. Systemic agents have been widely used for enhancing bone regeneration; however, the efficacy of these therapeutics for the management and prevention of fracture remains unclear. NEL-like protein 1 (NELL-1) is a potent pro-osteogenic cytokine that has been modified with polyethylene glycol (PEG)ylation [PEGylated NELL-1 (NELL-PEG)] to enhance its pharmacokinetics for systemic therapy. Our aim was to investigate the effects of systemic administration of NELL-PEG on fracture healing in mice and on overall bone properties in uninjured bones. Ten-week-old CD-1 mice were subjected to an open osteotomy of bilateral radii and treated with weekly injections of NELL-PEG or PEG phosphate-buffered saline as control. Systemic injection of NELL-PEG resulted in improved bone mineral density of the fracture site and accelerated callus union. After 4 weeks of treatment, mice treated with NELL-PEG exhibited substantially enhanced callus volume, callus mineralization, and biomechanical properties. NELL-PEG injection significantly augmented bone regeneration, as confirmed by high expression of bone turnover rate, bone formation rate, and mineral apposition rate. Consistently, the immunohistochemistry results also confirmed a high bone remodeling activity in the NELLPEG-treated group. Our findings suggest that weekly injection of NELL-PEG may have the clinical potential to accelerate fracture union and enhance overall bone properties, which may help prevent subsequent fractures. (Am J Pathol 2018, 188: 715-727; https://doi.org/10.1016/j.ajpath.2017.11.018)
\end{abstract}

Fracture healing is an intricate process that is heavily influenced by various systemic and local factors. ${ }^{1,2}$ The goal of fracture healing is to regain function and return to weight bearing in a timely manner. Improvements in overall skeletal strength may help prevent fractures, which are estimated to reach an incidence of 13.7 per 1000 adults annually. ${ }^{3}$ In fact, one in three older women and one in five older men will experience a fragility fracture, resulting in $>1.5$ million fragility fractures in the United States per year. ${ }^{4,5}$ With a growing aging population, the incidence of fragility fractures will increase and is projected to cost $\$ 25$ billion worldwide by 2025 , leading to a massive biomedical burden. ${ }^{6,7}$

Previously, studies on the therapeutic medical management of fractures have focused on parathyroid hormone, a
Food and Drug Administration-approved drug since 2002 for the treatment of osteoporosis. ${ }^{8,9}$ Reports of various animal models have shown the potential of teriparatide

\footnotetext{
Supported by NIH/National Institute of Arthritis and Musculoskeletal and Skin Diseases grants R01 AR066782-01 (K.T.), AR068835-01A1 (C.S.), and AR061399-01A1 (C.S.), an American Association of Orthodontists Foundation Orthodontic Faculty Development Fellowship award (J.H.K.), and National Center for Advancing Translational Science UCLA Clinical and Translational Science Institute (CTSI) grant UL1TR000124 (K.T.).

J.H.K., X.Z., B.W., C.S., and K.T. contributed equally as senior authors.

Disclosures: X.Z., B.W., K.T., and C.S. are inventors of NELL-1-related patents. X.Z., B.W., K.T., and C.S. are founders and/or previous board members of Bone Biologics Inc., which sublicenses NELL-1 patents from the University of California Regents, and also hold equity in the company.
} 
(parathyroid hormone 1-34) in accelerating fracture healing and preventing delayed unions; however, evidence from clinical trials has been insufficient to draw definitive conclusions. ${ }^{10}$ Nonetheless, teriparatide is still being used off label for management of fractures by physicians who are confident of its beneficial effect. ${ }^{8}$

Similarly, bisphosphonates (BPs), the most commonly used antiresorptive agents for osteoporosis therapy, have been shown to benefit patients to maintain bone mineral density (BMD) for up to 10 years after discontinuation. ${ }^{11,12}$ Current evidence regarding fracture risk reduction with prolonged BP therapy remains inadequate, ${ }^{13}$ and BP treatment does not appear to have a significant effect on fracture healing. ${ }^{14}$ Moreover, randomized clinical trials of the longterm use of BPs have been shown to induce osteonecrosis of the jaw, atypical femur fractures, and other adverse events, such as esophageal cancer, atrial fibrillation, and acute kidney injury. ${ }^{15}$ These dangerous adverse effects require clinicians to reevaluate treatment after 3 to 5 years to determine the relative benefits of continuation, discontinuation, or switching to another therapy. ${ }^{16,17}$

Normal fracture healing can restore the cellular composition and biomechanical function to the same level as its preinjury condition. ${ }^{18}$ Yet, delayed unions or nonunions do occur, with rates ranging from $1.7 \%$ to $18.5 \%,{ }^{19}$ leading to significant morbidity and disability. Emerging studies have shown improvement of fracture healing with local therapies in experimental models, ${ }^{18,20,21}$ but whether these treatments will translate to clinical settings remains unknown, particularly for systemic bone disorders that require systemic therapy for the management and prevention of fractures. ${ }^{22}$ Therefore, a novel systemic therapeutic strategy for augmentation of fracture healing and prevention of nonunion with fewer safety concerns is necessary.

NEL-like protein 1 (NELL-1) is a novel osteogenic molecule that has been extensively studied for its local bone regeneration potential on various animal models. ${ }^{23-26}$ Overexpression of NELL-1 results in premature fusion of cranial sutures in humans and mice, ${ }^{27}$ whereas Nell1-deficient mice exhibit impaired overall skeletal development and undermineralization. ${ }^{28}$ NELL-1 is a direct downstream effector of the osteogenic transcription factor Runt-related transcription factor- $2 .^{26}$ Mechanistically, it binds to the integrin $\beta 1$ receptor and activates the Wnt/B-catenin signaling pathway. ${ }^{29}$ Furthermore, NELL-1 is involved in the inhibition of adipogenesis through the peroxisome proliferator-activated receptor $\gamma$ signaling pathway ${ }^{30,31}$ and stimulates angiogenesis in pericytes at an early stage of bone regeneration. ${ }^{32}$ Local application of NELL-1 has had excellent results in bone augmentation in diverse animal models, such as calvarial defect, ${ }^{23}$ spinal fusion, ${ }^{33-35}$ and large femoral segmental defect. ${ }^{36}$ Recently, our group has successfully engineered NELL-1 with a Food and Drug Administration-approved technique of polyethylene glycol (PEG)ylation (NELL-PEG) for a systemic therapy for osteoporosis in ovariectomized mice. ${ }^{37,38}$ Despite the substantial evidence of NELL-1 in promoting osteogenesis, there has been little understanding of the effects of systemic delivery of NELL-1 on fracture healing.

In the present study, we explored the therapeutic effects of systemically delivered NELL-PEG on fracture repair in a radial defect model. We hypothesized that systemically delivered NELL-PEG improves fracture healing by enhancing bone regeneration and remodeling processes. The current study may present a new modality for the management and prevention of bone fracture, particularly in conditions of inherently weak bone, such as osteoporosis.

\section{Materials and Methods}

\section{Surgical Procedure and Animal Care}

A total of 10 female CD-1 mice, aged 10 weeks, were obtained from Charles River Laboratories (Wilmington, MA) and treated according to the animal protocol that was approved by the University of California, Los Angeles, Office of Animal Research Oversight and Chancellor's Animal Research Committee of the Office for Protection of Research Subjects. Animals were weighed and randomly assigned into control and treatment groups, then subjected to weekly tail vein injections of either PEGylated phosphate-buffered saline (PBS) or NELL-PEG ( $n=5$ animals per group). All surgical procedures were performed under anesthesia. The bilateral forelimbs were shaved and draped in a sterile manner before the surgery. An incision was made over the dorsum of the forearm, and the soft tissue was dissected bluntly to expose the radial shaft. The tip of an 11-blade scalpel was inserted into the interosseous membrane between the radius and ulna to protect the ulna, which was left intact. ${ }^{39}$ A $0.15-\mathrm{mm}$ Orthodisc (Ortho Technology, Tampa, FL) was used to generate an open osteotomy in the middle third of the radial shaft (Supplemental Figure S1A). The periosteum was removed and the skin incision was closed with 5-0 absorbable polyglactin sutures (Vicryl; Ethicon, Johnson \& Johnson, Somerville, NJ). Postoperatively, mice were housed individually with ad libitum access to food and water and allowed to weight bear as tolerated. Mice were weekly administered with $100 \mu \mathrm{L}$ of NELL-PEG solution $(1.25 \mathrm{mg} / \mathrm{kg})$ or PBS solution via the lateral vein, starting from the postsurgery (week 0 ) until the fourth injection (week 3 ), with the optimal dose determined according to our previous studies. ${ }^{37,38}$ Mice were sacrificed at week 4 postoperatively via carbon dioxide overdose and harvested for radii (Supplemental Figure S1B). Samples were fixed in 4\% paraformaldehyde for 48 hours and stored in $70 \%$ ethanol at room temperature.

\section{Radiographic Evaluation by Faxitron}

After the surgery, radiographs of the fracture site were taken immediately to confirm the transverse fracture. Subsequently, high-resolution radiographs were consecutively taken every 
week to monitor fracture healing. Three blinded examiners (J.T., J.K.K., and H.C.P.) assessed the healing process by using a 4-point radiograph-based scoring system from the radiographic union score. ${ }^{40}$ Fracture union was identified by the presence of bridging callus on two cortical edges.

\section{Dual X-Ray Absorptiometry Analysis}

All animals were subjected to dual X-ray absorptiometry scan (PIXImus2 GE Lunar Corp., Madison, WI) with an interval of every 2 weeks to monitor the changes in BMD. Scanning was performed under anesthesia. A square region of interest of $10 \times 10 \mathrm{~mm}^{2}$ was drawn at the fracture site. Longitudinal assessment of the BMD $\left(\mathrm{g} / \mathrm{cm}^{2}\right)$ was performed starting at the baseline until the final time point of the study.

\section{In Vivo MicroPET-CT Analysis}

At the end of the study, all animals were subjected to live micro-positron emission tomography-computed tomography (microPET-CT) scans at the University of California, Los Angeles, California NanoSystem Institute before harvest. Mice were preheated on a heating pad for 15 minutes before injection of $100 \mu \mathrm{Ci}$ of ${ }^{18} \mathrm{~F}-\mathrm{NaF}$ ion via tail vein injection and maintained under anesthesia on a heated induction chamber during the 1-hour tracer uptake, as stated previously. ${ }^{30,38,41}$ Briefly, all mice underwent 10 minutes of microPET scanning (Siemens Medical Solutions Inc., Knoxville, TN), followed by microCT scanning (Siemens Inveon CT). The filtered back projection of superimposed microCT radiographs on the reconstructed microPET images was used for analysis with A Medical Image Data Examiner software version 1.0.5 (https://en.freedown loadmanager.org/Mac-OS/AMIDE-FREE.html). A standardized region of interest was drawn in a box of $10 \times 10 \times 10$ $\mathrm{mm}^{3}$ to encompass the fracture site at the radii, whereas a box of $6 \times 6 \times 6 \mathrm{~mm}^{3}$ was drawn to cover the lower lumbar vertebrae (L6). Mean signal intensity (\% injected dose/g) of the tracer uptake was calculated, and values were quantified with decay correction and normalized according to the actual tracer-injected dose.

\section{Three-Dimensional MicroCT Analysis}

All specimens were scanned with SkyScan 1172 (Bruker microCT N.V., Kontich, Belgium), with settings of 55 $\mathrm{kVp}, 181 \mathrm{~mA}$, and $0.5-\mathrm{mm} \mathrm{Al} \mathrm{filter} \mathrm{at} \mathrm{the} \mathrm{resolution} \mathrm{of} 16$ $\mu \mathrm{m}$. Scanned images were then reconstructed with NRecon version 2.0.4 (Bruker microCT N.V.) for attenuation correction, ring artifact reduction, and beam hardening. Subsequently, samples were aligned in a three-dimensional view according to the long axis of the ulna with Data Viewer software version 1.5.4.0 (Bruker microCT N.V.). A total volume of interest of 200 slices was used for analysis, with the middle gap of the fracture as the midpoint reference. MicroCT images were analyzed with CTAn software version 1.13 (Bruker microCT N.V.) and segmented manually by comparing the binarized image with the unsegmented image. Threshold within the range of 60 to 225 was determined on the basis of subjective observation. The region of interest was manually drawn for improved accuracy by encompassing the callus and excluding the radius and ulna bones. Bone parameters of BMD $\left(\mathrm{g} / \mathrm{cm}^{3}\right)$, tissue mineral density, and trabecular thickness were used for analysis. CTVox software version 3.3 (Bruker microCT N.V.) was used to generate threedimensional representative images and videos. The linear measurement was performed with CTAn software version 1.17.7.2 by using a modified fracture displacement method. ${ }^{42}$ Most fractures were analyzed on the basis of the coronal or sagittal views of radiographs. Depending on the rotation of the object to the X-ray source, fracture displacement measurement was proved to be inconsistent unless a specific standardized protocol was established. ${ }^{43}$ Herein, we established a standardized protocol for measuring fracture displacement by calculating the shortest distance between fractured bones and the uninjured ulna on the two-dimensional transaxial images. The ulna was selected as a stable reference point.

\section{Biomechanical Testing by Finite Element Analysis}

Finite element models were generated by first converting microCT images into DICOM files using SkyScan Dicom Converter software version 2.5 (DicomCT application, SkyScan 1172F; Bruker microCT N.V.). Next, tetrahedral three-dimensional mesh models were generated by using Mimics software version 20.0 (Materialise, Leuven, Belgium) to isolate the volume of interest by drawing directly near the fracture site. The 3-matic version 12.0 (Materialise) was then used to remesh the models at an average of approximately 197,000 elements for each. Threepoint bending finite element analysis simulation ${ }^{44}$ was then performed using the ABAQUS software version 6.6 (Dassault Systèmes, Velizy-Villacoublay, France), with boundary conditions set as encastre at the most distal and proximal points. A Young's modulus of $3.5 \mathrm{GPa}$ and a Poisson's ratio of 0.33 were assigned to the radius and ulna. ${ }^{45}$ Subsequently, a uniform and uniaxial pressure of $0.5 \mathrm{MPa}$ was applied at the fracture site, to test the biomechanical strength of the healed bone. The average von Mises stress experienced by the samples ( $n=7$ limbs per group) was analyzed. For the analysis of the sixth lumbar vertebrate (L6), the same workflow was used to obtain the meshed models, except for the volume of interest, which was now selected to be a $0.6 \times 0.6 \times 0.6-\mathrm{mm}$ cube of trabeculae in the inferior portion of the vertebrae. On average, each meshed model is approximately 623,000 elements. In ABAQUS, a Young's modulus of $10 \mathrm{GPa}$ and a Poisson's ratio of 0.33 were assigned to simulate the material properties of the trabeculae bone. Similarly, a uniaxial 
compressive load of $0.5 \mathrm{MPa}$ was applied to the superior surface of the cube, whereas the inferior surface was set to encastre. The resulting average von Mises stress was then analyzed ( $n=5$ animals per group).

\section{Dynamic Bone Histomorphometric Analysis}

For bone fluorescent labeling, mice were injected intraperitoneally with Calcein $(20 \mathrm{mg} / \mathrm{kg})$ and Alizarin Red Complexon (30 mg/kg; Sigma Aldrich, St. Louis, MO) at weeks 1 and 3, respectively. After microCT scanning, samples were embedded in methylmethacrylate and processed for a longitudinal cut with a microtome $(n=3$ limbs per group). Bone double-labeling analysis was performed using the Osteomeasure Morphometric System version 3.0 (Osteometrics, Atlanta, GA), and parameters of mineral apposition rate ( $\mu \mathrm{m}$ per day) and bone formation rate $\left(\mu \mathrm{m}^{3} \mu \mathrm{m}^{-2}\right.$ per day) were used. The mineral apposition rate is the distance between the labels/the interval of injection time, whereas bone formation rate multiplies mineral apposition rate by the fraction of bone surface that is labeled. The analysis was performed at the fracture site as selected volume of interest. For each section, three consecutive images were taken at the fracture site for analysis.

\section{Histology and Immunohistochemistry Analysis}

Samples ( $n=7$ limbs per group) were soaked in a $19 \%$ EDTA solution for 14 days before cutting ( $5 \mu \mathrm{m}$ thick) at the Translational Pathology Core Laboratory, University of California, Los Angeles, Department of Pathology. All slides were used stained with hematoxylin and eosin, tartrate-resistant acid phosphatase (TRAP; Sigma Aldrich), osteocalcin (1:100; Santa Cruz Biotechnology, Dallas, TX), SRY-related HMG-box-9 (1:50; Santa Cruz Biotechnology), and CD-31/platelet endothelial cell adhesion molecule 1 (1:100; BioLegend, San Diego, CA) staining. Histologic assessment was performed based on Allen's scoring system for fracture healing. ${ }^{18}$ All samples were analyzed under the microscope, and three consecutive images at the fracture sites and adjacent area were captured using CellSense software version 1.12 (Olympus Corp., Tokyo, Japan).

\section{Blood Serum Marker Analysis by Enzyme-Linked Immunosorbent Assay}

An enzyme-linked immunosorbent assay was performed using blood serum samples, according to the manufacturer's recommendation. Blood samples were collected at weeks 0 , 2 , and 4 via the retro-orbital vein ( $n=5$ animals per group). Serum was collected and stored at $-80^{\circ} \mathrm{C}$ before analysis of serum procollagen I N-terminal propeptide and serum TRAP 5b (TRAP5b). All assays were obtained from ImmunoDiagnostic Systems Inc. (Boldon, UK).

\section{Statistical Analysis}

Standard descriptive statistics and 95\% CIs were estimated, and data were assessed for normal distribution. For longitudinal measurement, percentage change over time was calculated using a linear mixed model. Nonparametric data were analyzed with $U$ test when two groups were compared with each other. Unpaired $t$-test was used to compare means between the experimental and control groups. All analysis was performed by using the SPSS statistical package version 22.0 (IBM SPSS Statistics, Chicago, IL). Data are expressed as means $\pm \mathrm{SD} . P<0.5$ was considered statistically significant.

\section{Results}

\section{Systemically Delivered NELL-PEG Accelerates Callus Formation and Bridging}

Radiographs were taken weekly by Faxitron (Faxitron XRay Corporation, Lincolnshire, IL) and assessed by using a modified radiographic union scoring system ${ }^{40}$ (Table 1). Three blinded examiners (J.T., J.K.K., and H.C.P.) analyzed the radiographs. The intracorrelation coefficient was 0.873 for rater 1, 0.895 for rater 2, and 0.9 for rater 3. Meanwhile, the interrater reproducibility was 0.842 . Excitingly, at week 1 after surgery, 90\% (9 of 10) of the samples in the NELLPEG group started to develop callus adjacent to the fracture line; however, only half (5 of 10) of the PBS group had developed callus (Figure 1A). There was no significant difference in fracture healing found at week 2 because both groups showed apparent callus at the fracture area (Figure 1B). A substantial portion of the NELL-PEG (4 of 10) group has formed callus bridging at week 3 after surgery, whereas none of the PBS group had bridging callus at this time point. At the final time point, all of the samples (10 of 10) in the NELL-PEG group showed bridging callus, and $40 \%$ (4 of 10) had no visible fracture line (Figure 1B). The radiographs of the PBS group showed moderate callus formation, and $30 \%$ ( 3 of 10) of the samples failed to form a union at the end of the study (Figure 1, A and B).

\section{NELL-PEG Injection Improves Bone Density and Biomechanical Strength at the Fracture Site}

The results of biweekly dual X-ray absorptiometry measurement showed a gradual increase in BMD in both

Table 1 Radiographic Union Score

\begin{tabular}{ll}
\hline Score & Description \\
\hline 1 & Fracture line visible and no callus \\
2 & Fracture line visible and interrupted callus \\
3 & No fracture line, callus or fracture line visible, \\
& and continuous callus \\
4 & No fracture line and no callus \\
\hline
\end{tabular}


A
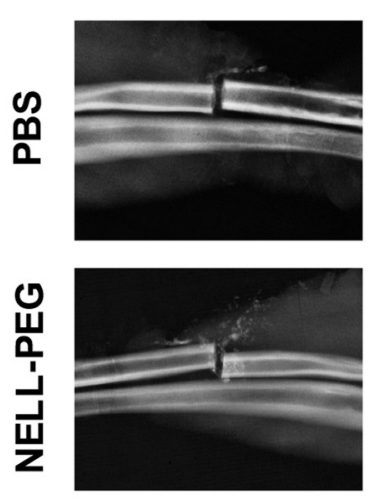

Week 1
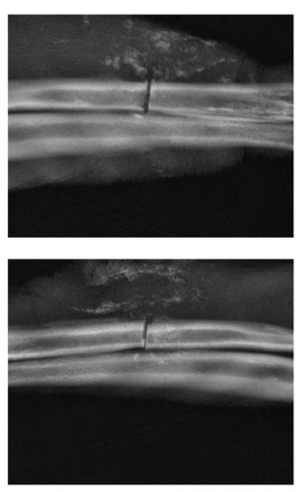

Week 2
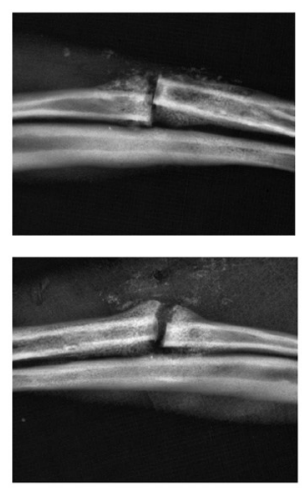

Week 3
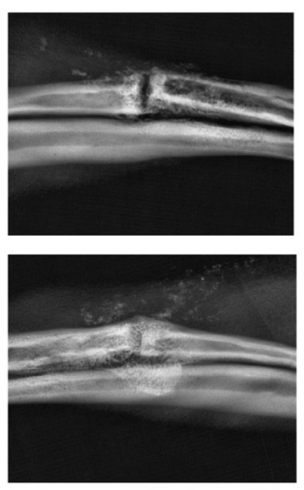

Week 4
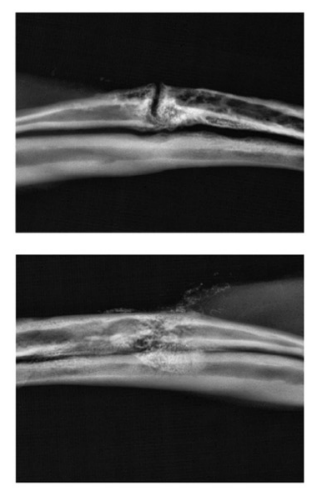

B

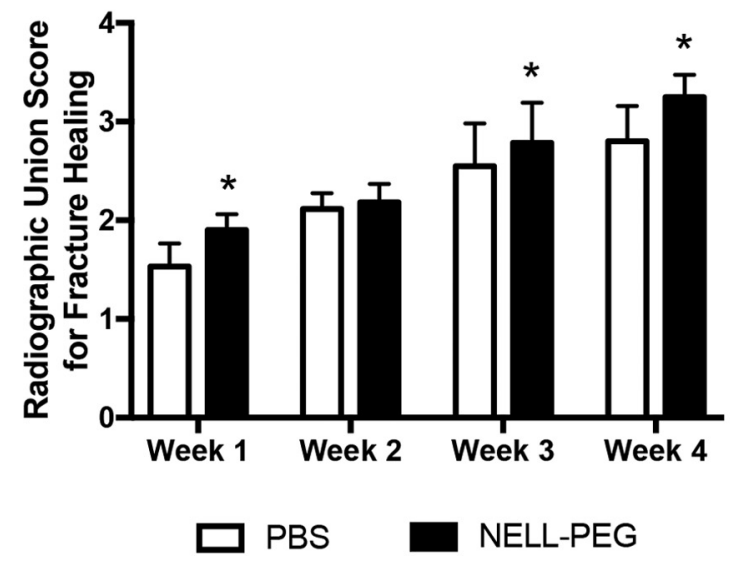

groups starting from week 2 until the end of treatment; the NELL-PEG group had a higher increase of BMD when compared with the PBS group. At week 2, the NELL-PEG group had significantly greater BMD at the fracture site compared with the PBS group. A similar trend was observed at week 4; the NELL-PEG group again showed significantly higher BMD (Figure 2A). We predicted the bone strength by performing a three-point bending biomechanical test ${ }^{44}$ on the microCT-derived finite element model (Figure 2B and Supplemental Video S1). The NELL-PEG group had enhanced bone strength and stiffness at the fracture site compared with the control group, as indicated by a significantly lower von Mises stress value (Figure 2, C and D).

\section{Systemic Treatment of NELL-PEG Increases Bone Formation and Bone Turnover Rate}

Bone dynamic histomorphometric analyses at the fracture site revealed significant increases of bone formation rate and mineral apposition rate (Figure 3, A-C). These findings are in agreement with the microPET-CT scans that demonstrated increased signal intensity in the fracture sites at the final time point, indicating a higher bone turnover rate at the
Figure 1 Weekly radiological assessment of fracture healing. A: Representative images of high-resolution radiographs of the phosphate-buffered saline (PBS) and NELL-PEG groups. Radiographs were taken every 7 days, starting immediately after surgery until the final time point of the study (week 4). B: Weekly radiographs were assessed for callus presence and bridging. The healing process was evaluated on the basis of the radiographic union score scoring system. ${ }^{40}$ Data are expressed as means \pm SD $(\mathbf{B}) .{ }^{*} P<0.05$. Original magnification, $\times 2.65(\mathrm{~A})$. fracture sites (Figure 3D). Furthermore, the NELL-PEG group also showed increased uptake of ${ }^{18} \mathrm{~F}-\mathrm{NaF}$ ions in the calvaria, in the axial skeleton, and around the growth plates of the appendicular bones (proximal humeri, distal femur, and proximal tibia), similar to previous observations. ${ }^{38,41}$ Quantification of the mean value at the fracture site exhibited a significantly higher tracer uptake in the NELL-PEG group compared with the PBS group (Figure 3E).

\section{NELL-PEG Treatment Enhances Bone Microarchitecture at the Fracture Site}

The NELL-PEG group exhibited a larger callus volume compared with the PBS callus at the final time point (Figure 4, A and E, and Supplemental Videos S2 and S3). Moreover, the NELL-PEG injection group had significantly higher BMD and tissue mineral density of the callus when compared with the PBS group (Figure 4, B and C). NELLPEG injection also enhanced the microarchitectural properties at the fracture site, as confirmed by a significantly higher trabecular thickness in the microCT analysis (Figure 4D). Interestingly, the NELL-PEG fractures were less displaced when the distance between fractured bone 
A

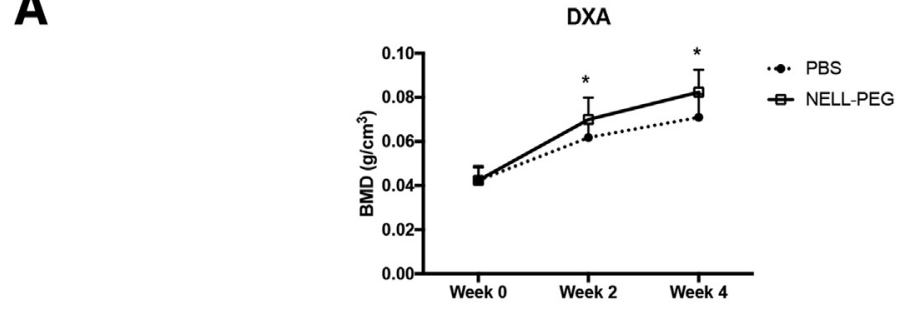

B

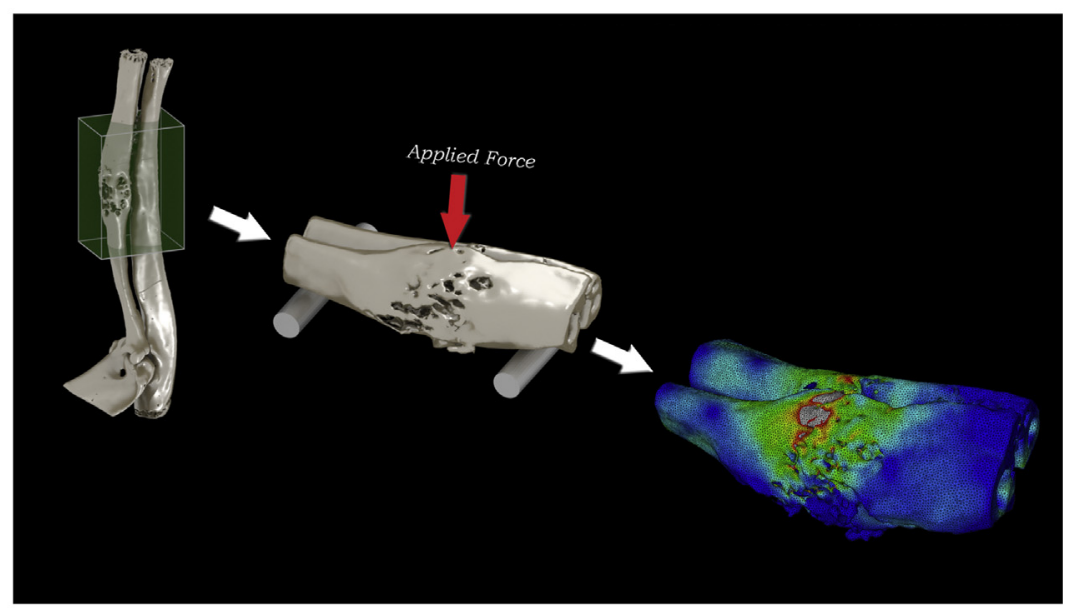

C

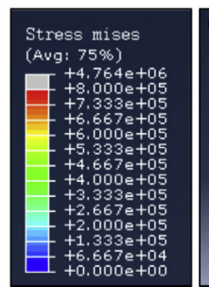

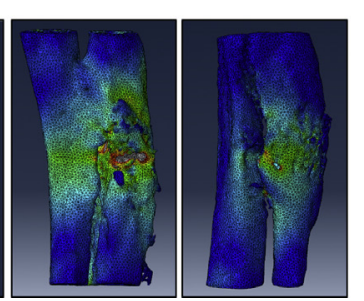

D

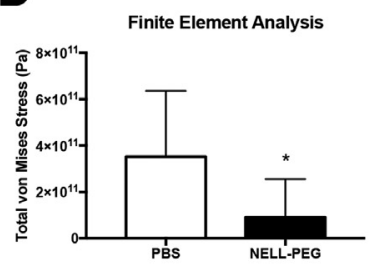

Figure 2 NELL-PEG injection improves bone density and biomechanical strength at the fracture site. A: Biweekly dual X-ray absorptiometry (DXA) measurement of phosphate-buffered saline (PBS) and NELL-PEG groups starting from week 0 (baseline) until week 4 . There is a gradual increase in bone mineral density (BMD) in both groups starting from week 2 toward the end of the treatment; at week 2, the NELL-PEG group has significantly greater BMD at the fracture site compared with the PBS group. A similar trend is observed at week 4, when the NELL-PEG group shows significantly higher BMD compared with the PBS group. B: Biomechanical strength prediction by a threepoint bending test on a microCT-derived finite element model. C: Representative images of finite element analysis. Red indicates areas of high stress. D: Quantification of total von Mises stress $(\mathrm{Pa})$ value. Data are expressed as means \pm SD (A and $\mathbf{D}) .{ }^{*} P<0.05$. edges and the uninjured ulna was calculated using a modified fracture displacement method ${ }^{42}$ (Figure 4, F and G).

\section{NELL-PEG-Treated Callus Has Elevated Bone Remodeling Activity alongside with Enhanced Angiogenesis}

Histologic results were assessed using the Allen's scoring system of fracture healing (Table 2). The results were concordant with imaging in that all of the samples in the NELL-PEG group showed more robust healing and bigger callus at the fracture site (Figure 5A). In agreement with the radiographic results, some samples in the PBS group (three of seven) did not show a bony union because of some fibrous remnants within the callus at the end of the study (Figure 5, A and B). The osteogenic effects of NELL-PEG treatment on fracture healing were confirmed by immunohistochemistry analysis (Figure 6, A-D). Staining of an osteoblast marker, osteocalcin, was increased in the NELLPEG group, whereas an osteoclast marker, TRAP, was decreased (Figure 6, A and B). In addition, the early cartilaginous chondrogenic marker SRY-related HMG-box-9 was tested, and the expression was elevated in the PBS group as opposed to the NELL-PEG group, which had no remnants of cartilaginous bone (Figure $6 \mathrm{C}$ ). Last, to evaluate the effect on angiogenesis of systemically delivered NELL-PEG, an immunohistochemistry staining of the CD31/platelet endothelial cell adhesion molecule 1 marker was performed. The NELL-PEG group exhibited a higher expression of CD-31/platelet endothelial cell adhesion molecule 1-positive staining compared with the PBS group (Figure 6D).

\section{Systemic Effects of NELL-PEG on Overall Bone Properties}

To further test the systemic effects of NELL-PEG on overall uninjured bone properties, the lower lumbar vertebrae region was analyzed. Consistent with reported findings, ${ }^{30,38}$ microCT analysis at the L6 area revealed significant increases in BMD and bone volume/tissue volume in the NELL-PEG group compared with the PBS group (Figure 7, $\mathrm{A}-\mathrm{C}$ ). Furthermore, serum biomarker tests revealed a significant increase of bone formation marker serum 
A
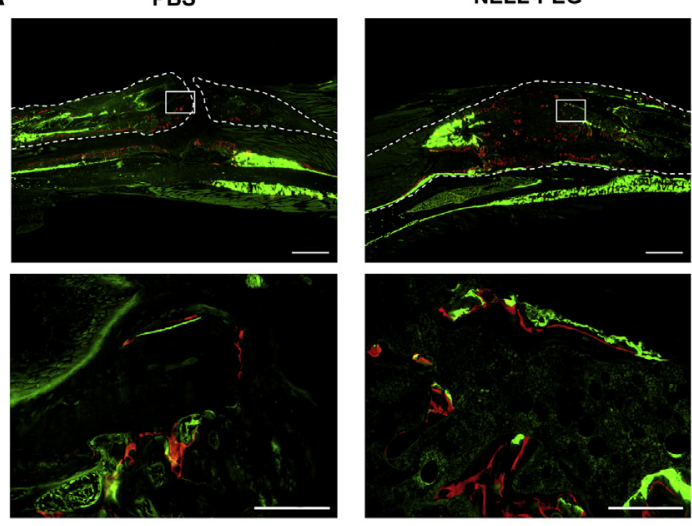

D

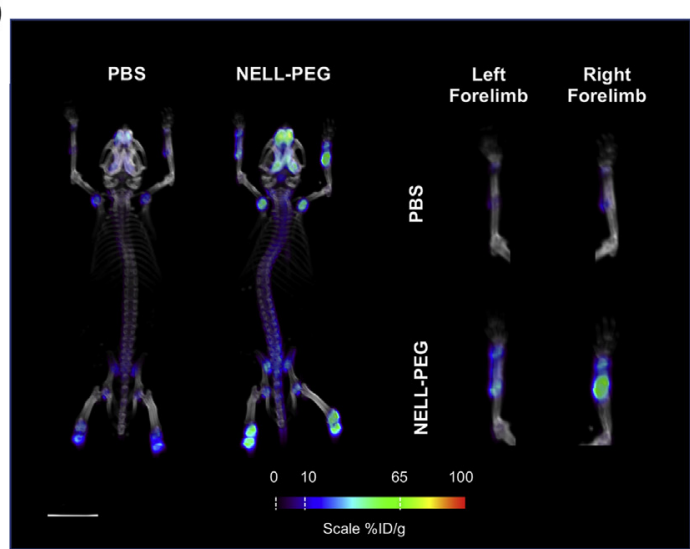

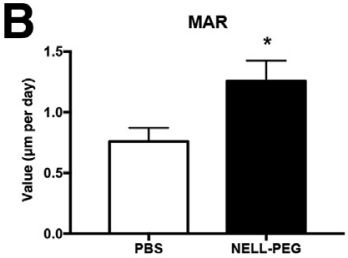

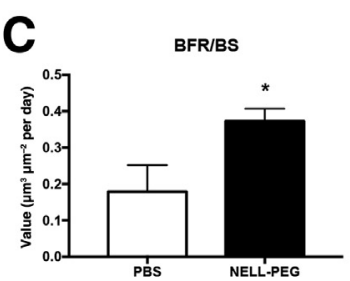

E

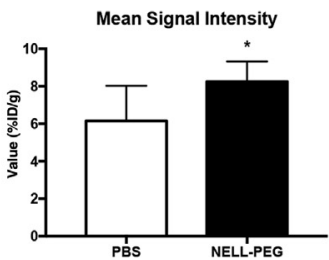

Figure 3 Systemic treatment of NELL-PEG increases bone formation and bone turnover rate. A: Representative images of dynamic bone labeling of Calcein (week 1) and Alizarin Red Complexon (week 3) at the fracture site. The phosphatebuffered saline (PBS) group shows nonunion callus with less Alizarin label deposited at the fracture site, whereas the NELL-PEG group shows a high intensity of bone labels deposited within the callus. Dashed lines represent the cortical border of radius. Boxed areas in the top row represent midcallus areas, which are shown at higher-power magnification in bottom row. B and C: Quantification of bone labeling histomorphometric analysis reveals significant increases of mineral apposition rate (MAR) and bone formation rate (BFR). D: Representative images of bone turnover rate assessment by the microPET-CT scan after injection of the ${ }^{18} \mathrm{~F}-\mathrm{NaF}$ ion. The NELL-PEG group shows increased uptake of the tracer at the fracture site compared with the PBS group. E: Quantification of the mean value ratio at the fracture site exhibits significantly higher tracer uptake in the NELL-PEG group compared with the PBS group. Data are expressed as means \pm SD $(\mathbf{B}, \mathbf{C}$, and $\mathbf{E})$. ${ }^{*} P<0.05$. Scale bars: $8 \mathrm{~mm}(\mathbf{A}$, top row, and $\mathbf{D})$; $16 \mathrm{~mm}$ (A, bottom row). Original magnification: $\times 2$ ( A, top row); $\times 20$ (A, bottom row). BS, bone surface; ID, injected dose. procollagen I N-terminal propeptide, particularly at week 4 of treatment. Serum procollagen I N-terminal propeptide marker was slightly increased at weeks 2 and 4 in the NELL-PEG group compared with the PBS group, which exhibited a decrease of serum procollagen I N-terminal propeptide toward the end of the treatment. Bone resorption marker serum TRAP5b showed a marked decrease at weeks 2 and 4 in the NELL-PEG group, whereas the PBS group showed a slight increase at week 2 and a decline at week 4 . At week 2, the NELL-PEG group revealed a significant difference in the serum TRAP5b levels compared with the PBS group (Figure 7, D and E). MicroPET-CT analysis of the tracer uptake at the lower lumbar vertebrae (L6) exhibited significantly higher tracer uptake in the NELLPEG group compared with the PBS group (Figure 7F). Consistent with these findings, the finite element analysis at the L6 revealed the NELL-PEG group had a significantly lower average Von Mises stress compared with the PBS group (Figure $7 \mathrm{G}$ ).

\section{Discussion}

Fracture healing can be enhanced by several strategies, including local or systemic therapeutics and biophysical enhancements. ${ }^{1}$ Studies of local interventions to accelerate fracture healing have been burgeoning recently, inclusive of growth factors, scaffolds, implant coatings, and injectable mesenchymal stem cells. ${ }^{20,21}$ Although local therapies allow the therapeutic agents to induce direct and rapid effects on the targeted region, these strategies are associated with adverse effects, ${ }^{46,47}$ limitations, ${ }^{4-51}$ and high costs, ${ }^{48}$ as opposed to systemic therapeutics, which offer a noninvasive stimulation of bone fracture healing for a prolonged period of time, especially in cases of systemic bone disorders causing multiple fractures. ${ }^{52-54}$ Systemic agents have been widely used in the management of fractures, especially in osteoporotic patients who have a greater risk of fractures because of a significant reduction of BMD. 9,22,55 Both anabolic and antiresorptive agents have been extensively studied for their effects on fracture prevention and fracture healing, yet fragility fractures with delayed and nonunion still occur in patients receiving therapeutic intervention. ${ }^{19,52}$ For instance, long-term use of BPs may induce abnormal bone remodeling because of depletion of osteoclast activity. Therefore, there is an acute concern for nonunion in patients receiving BPs. ${ }^{56}$ With these limitations of current therapeutics, NELL-1 may offer a new strategy as a systemic therapeutic enhancement for fracture healing, especially in populations at elevated risk for delayed or nonunion.

During the past decades, NELL-1 has been widely studied for its local and systemic application, particularly in the 


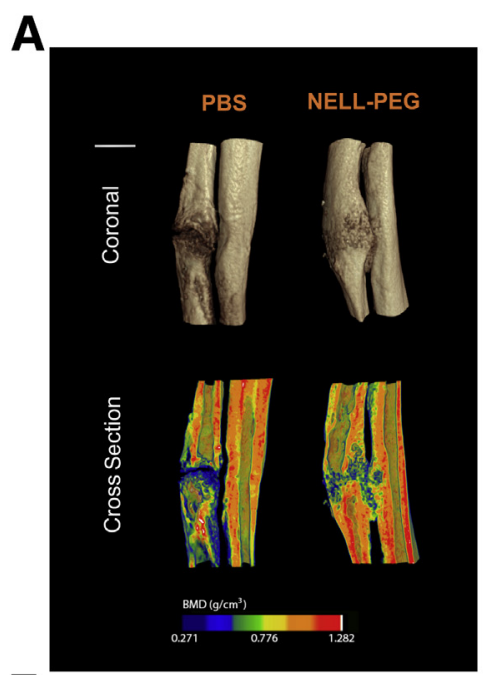

$\mathbf{F}$

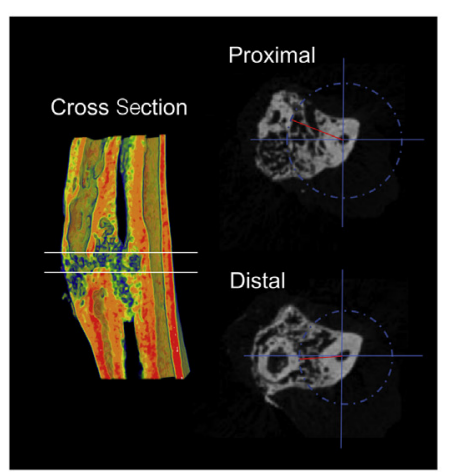

B

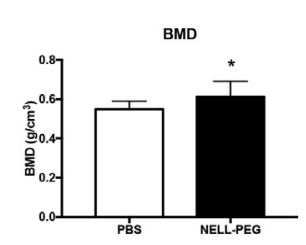

D

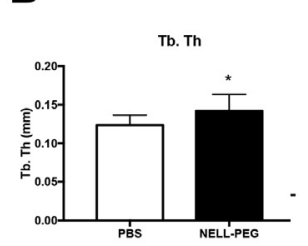

G

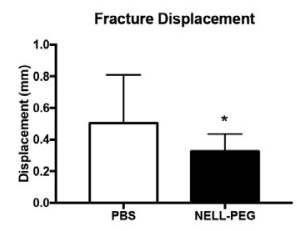

C

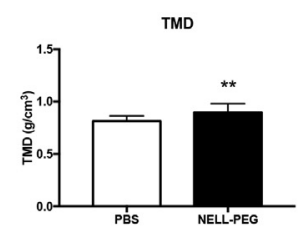

E

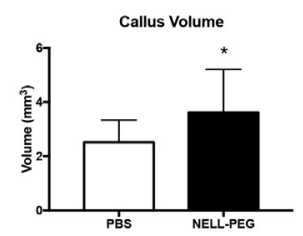

Figure 4 MicroCT analysis of fracture healing. A: Representative three-dimensional microCT images of phosphate-buffered saline (PBS) and NELL-PEG groups at week 4. B and C: The NELL-PEG injection group has a significantly higher bone mineral density (BMD) and tissue mineral density (TMD) of callus compared with the PBS group. D: The trabecular thickness ( $\mathrm{Tb}$. Th) at the fracture site is significantly higher in the NELL-PEG group compared with the control group. E: Total volume of the callus in the NELL-PEG group is substantially higher than in the PBS group. F: Linear measurement on a twodimensional microCT transaxial view. The top solid line represents the proximal view, and the bottom solid line represents the distal view. Region of interest is represented by the area between two solid lines. The blue compass represents the range, whereas the red line represents the linear measurement of the closest distance from ulna to the tip of fractured radius. G: Quantification of displacement of the fractured bone edges in PBS and NELL-PEG groups. NELL-PEG group shows significantly shorter distance compared with the PBS group when the distance from the fractured bones to the uninjured ulna is measured. Data are expressed as means \pm SD (B-E and $\mathbf{G}) .{ }^{*} P<0.05$, $* * P<0.01$. Scale bar $=1 \mathrm{~mm}(\mathbf{A})$.

context of osteoporosis. As opposed to other Wnt/ $\beta$-catenin regulators, NELL-1 exerts its effects on both osteoblasts and osteoclasts, representing a dual-effect pharmacotherapy agent. ${ }^{30}$ Moreover, in vivo toxicity test of 5-day consecutive injections of high doses of NELL-1 $(6.25 \mathrm{mg} / \mathrm{kg})$ via lateral tail vein did not show any pathology in the vital organs. Recently, our group has successfully enhanced the pharmacokinetics of NELL-1 by using a Food and Drug Administration-approved method of attaching a watersoluble PEG to the protein. ${ }^{57}$ With PEGylation, the halflife of NELL-1 was improved by threefold without any considerable toxicity ${ }^{37,38}$; thus, NELL-1 is reasonably safe for systemic application. In the present study, we investigated the systemic effects of a weekly low-dose injection $(1.25 \mathrm{mg} / \mathrm{kg})$ of NELL-PEG in a mouse radial defect model that left the ulna intact for partial stabilization; therefore, nonunion may be achieved by this unstable fixation model. Depending on the movement at the fracture site, fracture healing may result in heterotrophic nonunion or delayed union. ${ }^{58}$ To this point, the appropriate size of the defect in murine radius fracture model that leads to nonunion at 28 days has yet to be defined. ${ }^{58}$ The findings from the pilot study had suggested that a $0.15-\mathrm{mm}$ defect may produce a nonunion and the defect size is easily reproducible.

Typically, the healing process of a nonfixated fracture will promote secondary bone remodeling through a combination of intramembranous and endochondral ossification, with more cartilaginous bone formed in a less stable fracture. ${ }^{59}$ This process is tightly regulated by the Wnt signaling in a stage-specific manner. ${ }^{60}$ In early endochondral bone repair, $\beta$-catenin regulates the relationship between the number of osteoblasts and chondrocytes that arise from pluripotent mesenchymal cells. Later during the process, $\beta$-catenin promotes the differentiation of osteoblasts and promotes bone matrix production; in the remodeling phase, a slight activation of $\beta$-catenin may result in enhanced bone strength. ${ }^{60}$ Next, the catabolic phase occurs, characterized by active resorption of excess callus. ${ }^{61}$ These findings are consistent with the results that NELL-PEG-treated samples expressed a higher osteoblastic activity with reduced osteoclastic activity within the callus, which is similar to reported findings. ${ }^{34,36,41,62}$ Previously, NELL-1 was found to induce mesenchymal stem cell expansion ${ }^{36}$

Table 2 Allen's Classification of Fracture Healing

\begin{tabular}{ll}
\hline Grade & Description \\
\hline 0 & Pseudoarthrosis formation \\
1 & Incomplete cartilaginous union \\
2 & Complete cartilaginous union \\
3 & Incomplete bony union \\
4 & Complete bony union \\
\hline
\end{tabular}


A
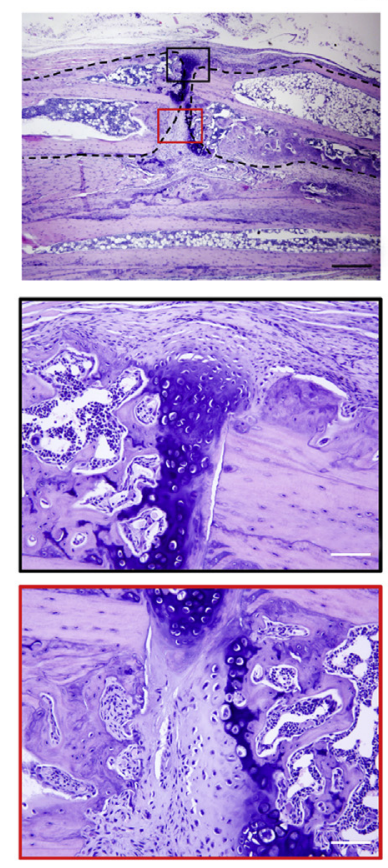

PBS
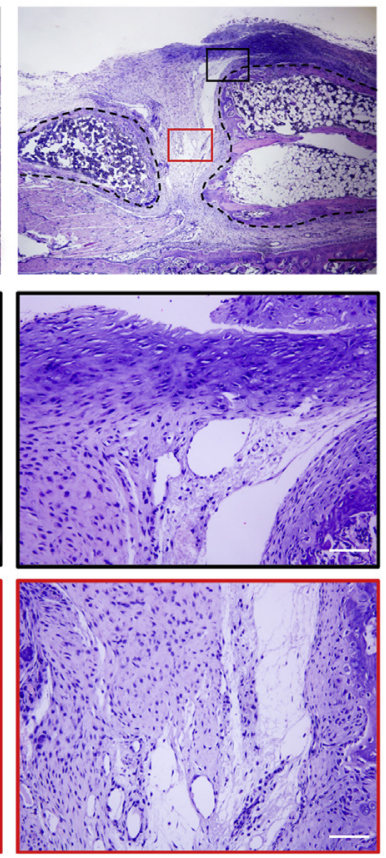

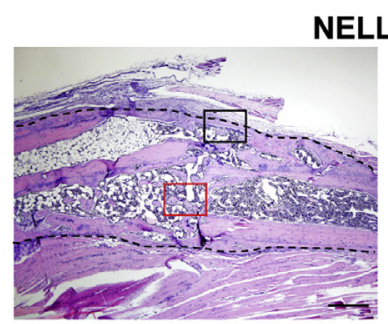

NELL-PEG
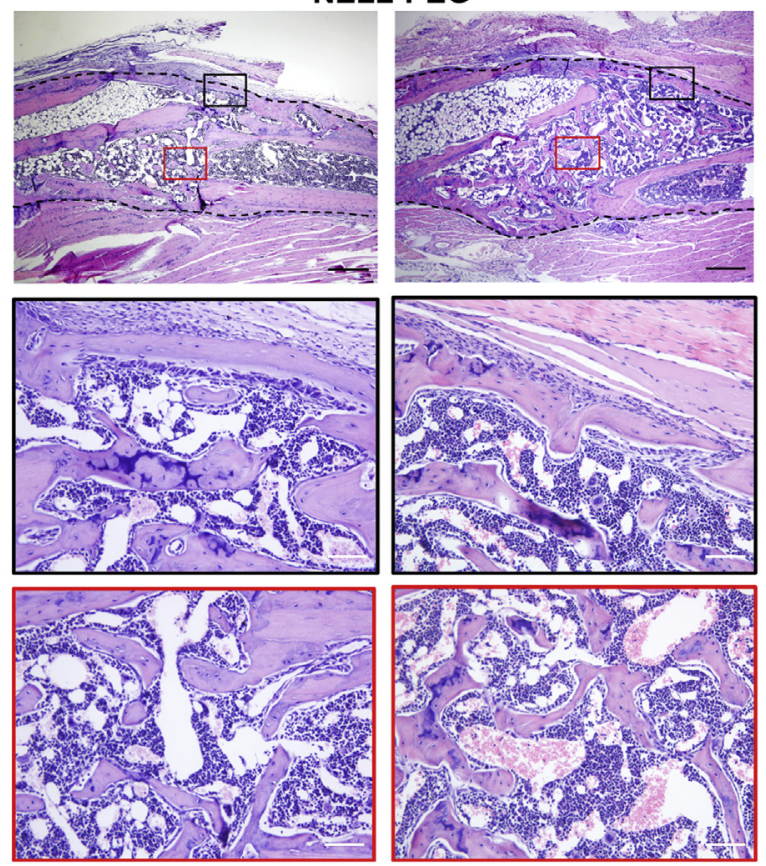

\section{B}

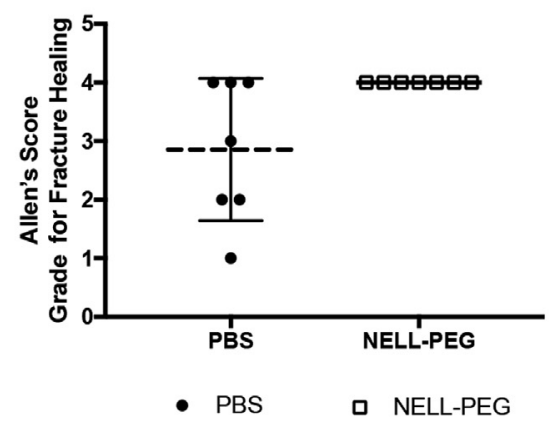

Figure 5 Histologic findings. A: Hematoxylin and eosin staining of the fracture site and adjacent area in both phosphate-buffered saline (PBS) and NELL-PEG groups. At the final time point of the treatment (week 4), PBS group shows nonunion of the callus with fibrocartilaginous remnants at the fracture site. Dashed lines represent the cortical border of radius. Black boxed areas denote perifracture areas, and red boxed areas denote midcallus areas. Boxed areas in top panels are shown at higher power magnification in middle and bottom panels. B: Histologic assessment was based on Allen's scoring system for fracture healing. ${ }^{18}$ The PBS group shows three of seven samples fail to form a union at week 4, whereas all samples in the NELL-PEG group are healed. Original magnification: $\times 4$ (A, top panels); $\times 20$ (A, middle and bottom panels). and osteogenic differentiation during bone repair. ${ }^{62}$ In the spinal fusion model, recombinant human NELL-1 protein release resulted in a local influx of stem cell antigen1 -positive mesenchymal progenitor cells. ${ }^{35}$ Collectively, these findings suggest that NELL-1 affects the stem cell niche, supporting osteoblastic differentiation during the initiation of fracture healing.

Interestingly, the NELL-PEG group has increased osteoclastic activity at the lining of the callus area at week 4 , even though the TRAP5b blood serum marker remained at a low level. In contrast, the PBS group did not show any osteoclast activity at the fracture site, whereas the TRAP5b marker showed high activity at week 2 and decreased almost to the same level as NELL-PEG at week 4. The osteoclasts in the NELL-PEG group may be activated by other factors during the remodeling phase to complete the fracture repair, which allows the callus to be actively resorbed until it is reshaped into its original anatomy. Although the anabolic and antiresorptive effects of NELL-1 have been well established in the previous animal models, ${ }^{30,37,38}$ the regulation of NELL-1 on osteoblasts and osteoclasts at different phases of bone healing has not been examined in vivo. Overall, we demonstrated NELL-PEG as a promising fracture therapy with a dual function of anabolic and antiresorptive properties that has the potential to accelerate fracture healing.

In addition, the NELL-PEG group had minimal chondrocyte (SRY-related HMG-box-9) expression compared with the PBS group, suggesting that the NELL-PEG group did not have any fibrocartilaginous remnants in the callus area at the terminal time point. Moreover, CD-31 expression of matured endothelial cells for angiogenesis showed that the NELL-PEG group had more vascularization in the fracture site and the bone lining matrix as opposed to the PBS group, which did not show any active vascularization. Studies of devascularization of the blood supply with electrocautery have been shown to produce nonunion in the mouse femur fracture model ${ }^{63,64}$; therefore, an appropriate 
A
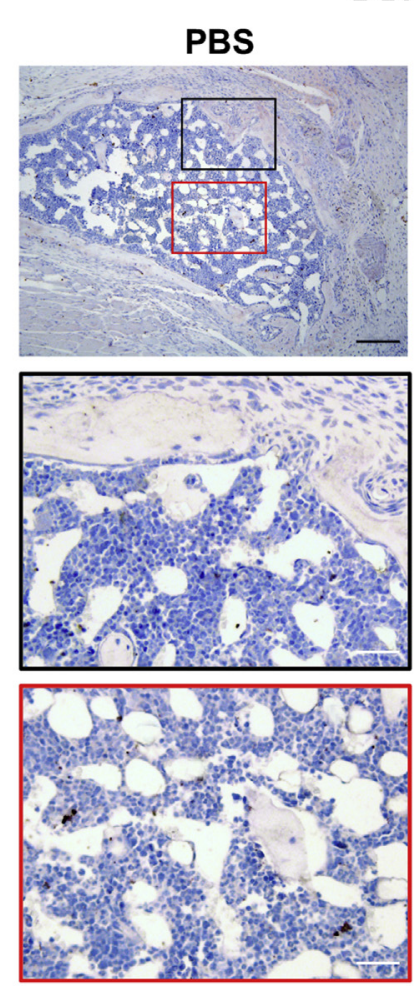

C
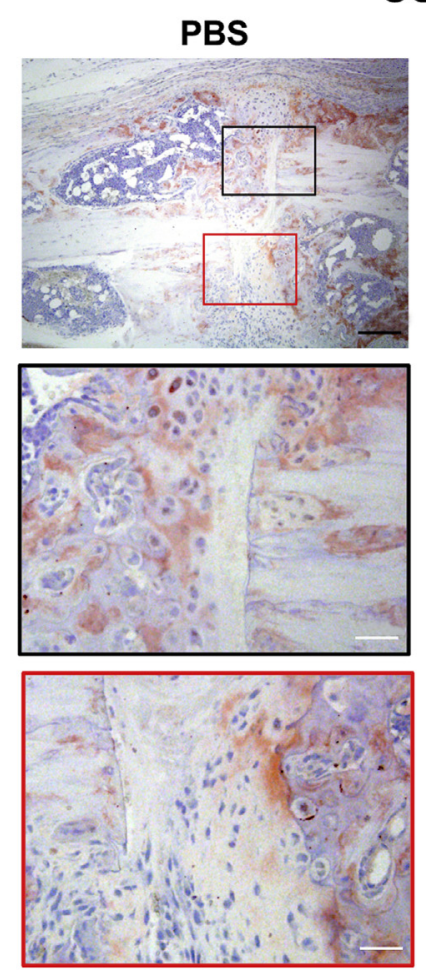

OCN
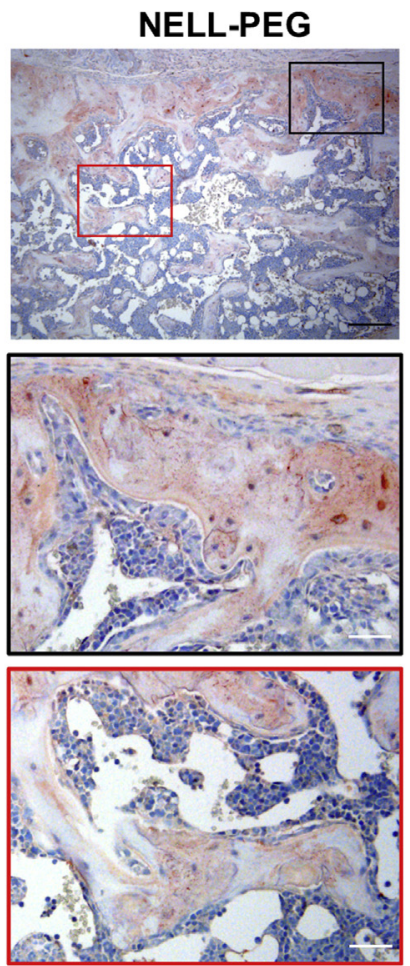

SOX9
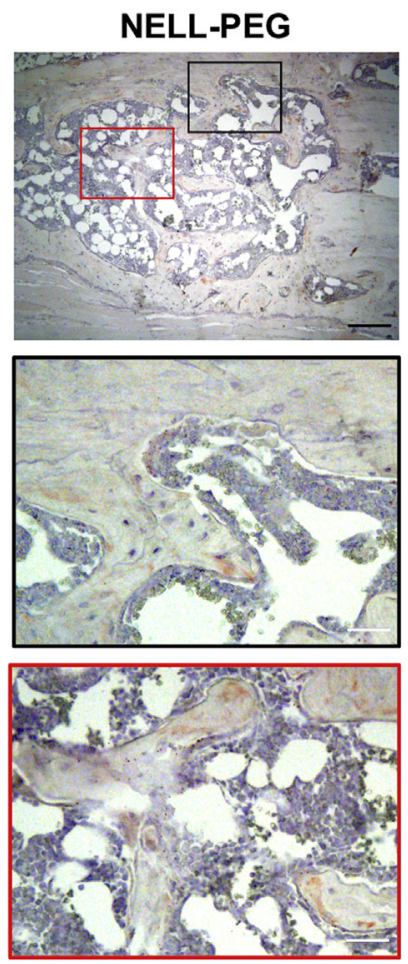

B
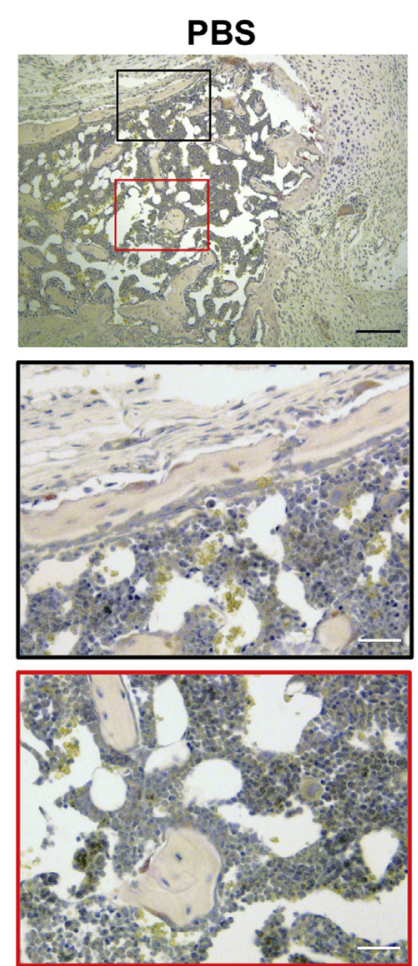

D

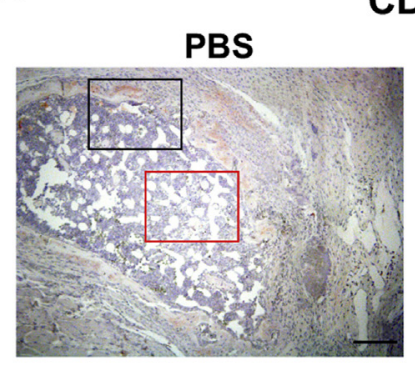

CD31
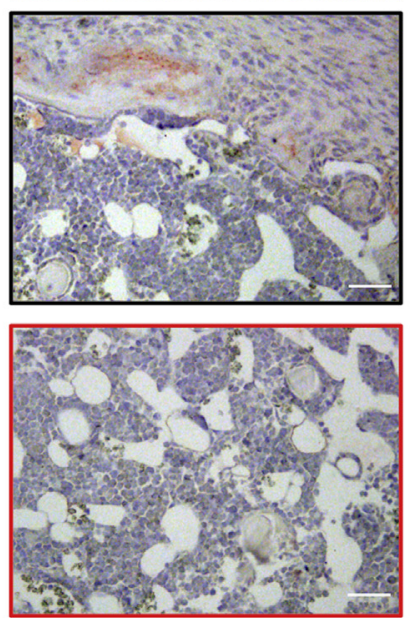
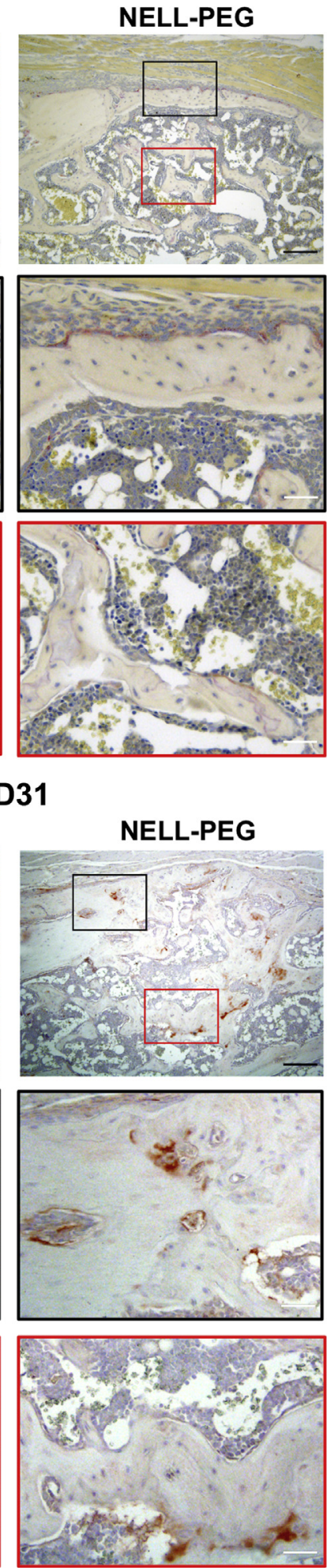

Figure 6 Immunohistochemistry analyses. A-D: Staining of osteocalcin (OCN), tartrate-resistant acid phosphatase (TRAP), SRY-related HMG-box-9 (SOX9), and CD-31/platelet endothelial cell adhesion molecule 1 (CD31). A: NELL-PEG group shows increased osteoblastic activities and decreased osteoclastic activities in the callus area. B: The lining of the bony callus of NELL-PEG group exhibits some TRAP-positive cells, but they are not found in the phosphatebuffered saline (PBS) group. C: SOX9 marker is elevated in the PBS group as opposed to the NELL-PEG group, which has no remnants of cartilaginous bone. D: The NELL-PEG group exhibits a higher expression of CD31-positive staining compared with the PBS group. Black boxed areas show perifracture areas, and red boxed areas show midcallus areas. Boxed areas in top panels are shown at higher power magnification in middle and bottom panels. Original magnification: $\times 10$ (A-D, top panels); $\times 20$ (A-D, middle and bottom panels). 


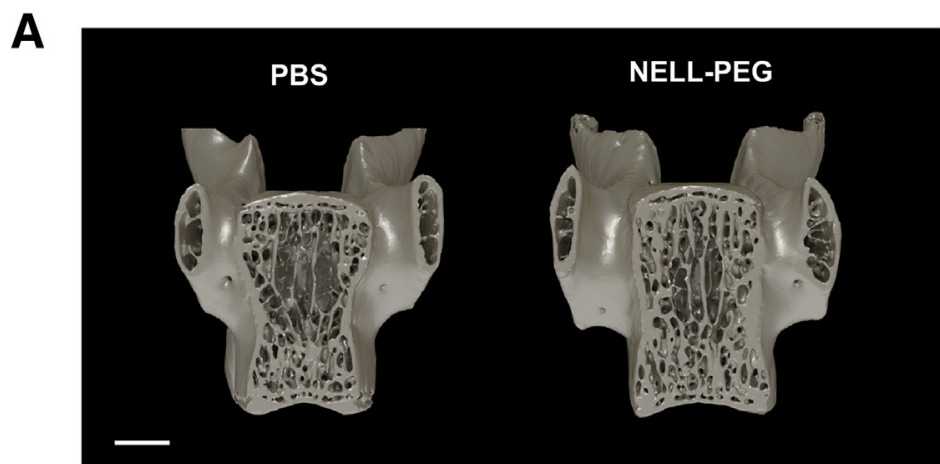

B

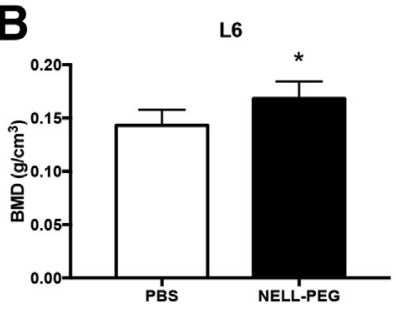

D
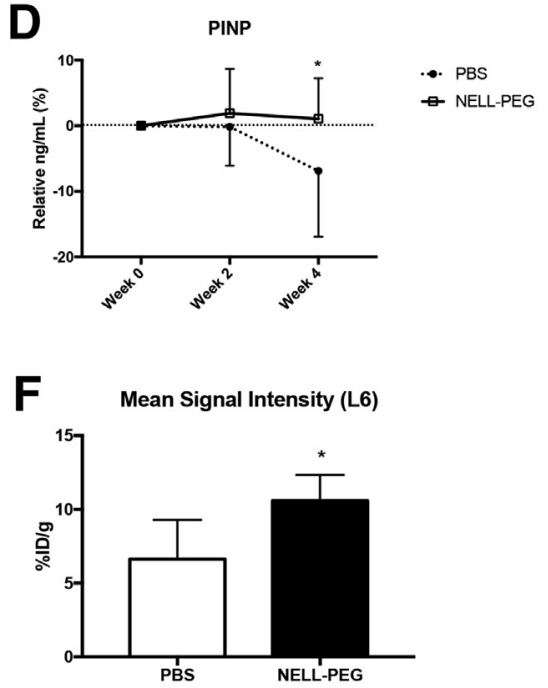

C

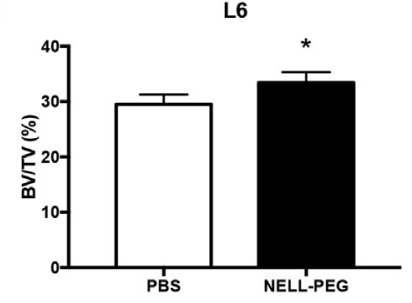

E

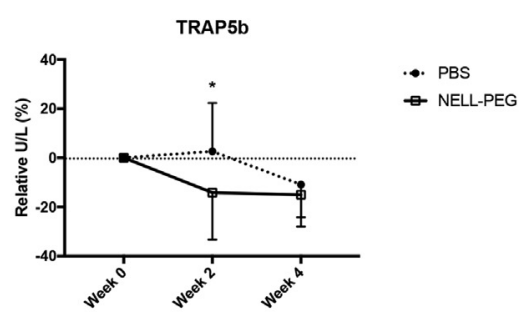

G

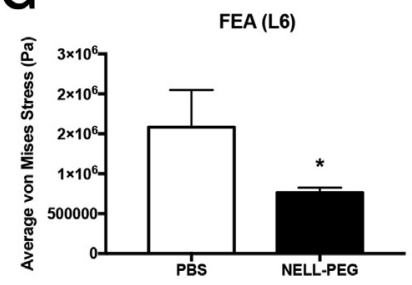

Figure 7 Systemic effects of NELL-PEG on overall bone properties. A: Three-dimensional view of lower lumbar vertebra (L6). B: Analysis of microCT reveals a significant increase of bone mineral density (BMD) value in the NELL-PEG - treated group. C: The bone volume/tissue volume (BV/TV) is significantly increased in the NELL-PEG group as opposed to the phosphate-buffered saline (PBS) group. D: Serum procollagen I N-terminal propeptide (PINP) marker is slightly increased at weeks 2 and 4 in the NELL-PEG group compared with the PBS group, which exhibits a decrease of serum PINP toward the end of the treatment. At week 4, the PBS group shows a significantly lower serum PINP level compared with the NELL-PEG group, in which the level remains plateaued throughout the study. E: Serum tartrate-resistant acid phosphatase 5b (TRAP5b) shows a marked decrease at weeks 2 and 4 in the NELL-PEG group, whereas the PBS group shows a slight increase at week 2 and a decline at week 4. At week 2, the serum TRAP5b in the NELLPEG group is significantly lower than in the PBS group. F: MicroPET-CT quantification of the mean value ratio at lower lumbar vertebrae (L6). The results exhibit significantly higher tracer uptake in the NELL-PEG group compared with the PBS group. G: Finite element analysis at the L6 reveals the NELLPEG group has a significantly lower average von Mises stress compared with the PBS group. Data are expressed as means $\pm S D(B-G)$. Scale bar $=1 \mathrm{~mm}$ (A). ${ }^{\star} P<0.05$. ID, injected dose. blood supply is also an essential component of fracture healing. ${ }^{18}$ Last, systemically delivered NELL-PEG has an active anabolic effect on the uninjured bone site of the lower lumbar vertebrae region, one of the most common sites of osteoporotic fractures. ${ }^{65}$ The BMD and bone volume/tissue volume were significantly increased in the L6 vertebra, which is consistent with reported findings. ${ }^{30,38,41}$ Similarly,

${ }^{18} \mathrm{~F}-\mathrm{NaF}$ incorporation of the microPET scan showed that NELL-PEG injection also increased the bone turnover rate in the lower lumbar vertebrae region (L6). Thus, NELLPEG may enhance whole-body BMD and even prevent future fractures.

This is the first study that examines the effects of systemic administration of NELL-PEG on fracture healing. We have demonstrated the ability of systemically delivered NELL-PEG to accelerate fracture healing via enhancement of bone regeneration and remodeling processes. More important, NELL-PEG also exerts its positive effects of enhancing bone microarchitecture not only at the fracture site but also on the overall skeleton, most notably the bone sites that are prone to fragility fractures. From a clinical perspective, these findings suggest that NELL-1 has immense potential to be developed as a future strategy for the treatment of fractures. In addition to its osteogenic properties, NELL-1 has also been applied as a potent systemic osteoporotic agent in various animal models with great success; therefore, future studies will aim to explore the therapeutic potential of NELL-1 in enhancing fracture healing in the age-related bone diseases (namely, osteoporosis). Beyond fragility fractures, there may also be applications in delayed unions, nonunions, and fractures in patients with known risk factors for delayed healing. Furthermore, there is potential for acceleration of normal fracture healing, which may lead to earlier initiation of 
weight bearing in fractured limbs. The unique properties of NELL-1 as a dual therapeutic agent with an antiadipogenesis effect, as well as the low toxicity in mice, make it a promising candidate for future clinical application in the prevention and treatment of fractures.

\section{Acknowledgments}

We thank the University of California, Los Angeles, Translational Pathology Core Laboratory and the California NanoSystems Institute for expertise; we also thank Dr. Renata Pereira, Dr. Pin Ha, Dr. Wenlu Jiang, and Ji E. Shin for their contributions.

\section{Supplemental Data}

Supplemental material for this article can be found at https://doi.org/10.1016/j.ajpath.2017.11.018.

\section{References}

1. Einhorn TA, Gerstenfeld LC: Fracture healing: mechanisms and interventions. Nat Rev Rheumatol 2015, 11:45-54

2. Marsell R, Einhorn TA: The biology of fracture healing. Injury 2011, 42:551-555

3. Court-Brown CM, Caesar B: Epidemiology of adult fractures: a review. Injury 2006, 37:691-697

4. Becker DJ, Kilgore ML, Morrisey MA: The societal burden of osteoporosis. Curr Rheumatol Rep 2010, 12:186-191

5. Eisman JA, Bogoch ER, Dell R, Harrington JT, McKinney RE Jr, McLellan A, Mitchell PJ, Silverman S, Singleton R, Siris E; ASBMR Task Force on Secondary Fracture Prevention: Making the first fracture the last fracture: ASBMR task force report on secondary fracture prevention. J Bone Miner Res 2012, 27:2039-2046

6. Burge R, Dawson-Hughes B, Solomon DH, Wong JB, King A, Tosteson A: Incidence and economic burden of osteoporosis-related fractures in the United States, 2005-2025. J Bone Miner Res 2007, 22:465-475

7. Solomon DH, Johnston SS, Boytsov NN, McMorrow D, Lane JM, Krohn KD: Osteoporosis medication use after hip fracture in U.S patients between 2002 and 2011. J Bone Miner Res 2014, 29: 1929-1937

8. Babu S, Sandiford NA, Vrahas M: Use of teriparatide to improve fracture healing: what is the evidence? World J Orthop 2015, 6: 457-461

9. Kim SM, Kang KC, Kim JW, Lim SJ, Hahn MH: Current role and application of teriparatide in fracture healing of osteoporotic patients: a systematic review. J Bone Metab 2017, 24:65-73

10. Shi Z, Zhou H, Pan B, Lu L, Liu J, Kang Y, Yao X, Feng S: Effectiveness of teriparatide on fracture healing: a systematic review and meta-analysis. PLoS One 2016, 11:e0168691

11. Black DM, Schwartz AV, Ensrud KE, Cauley JA, Levis S, Quandt SA, Satterfield S, Wallace RB, Bauer DC, Palermo L, Wehren LE, Lombardi A, Santora AC, Cummings SR; FLEX Research Group: Effects of continuing or stopping alendronate after 5 years of treatment: the Fracture Intervention Trial Long-term Extension (FLEX): a randomized trial. JAMA 2006, 296:2927-2938

12. Schwartz AV, Bauer DC, Cummings SR, Cauley JA, Ensrud KE, Palermo L, Wallace RB, Hochberg MC, Feldstein AC, Lombardi A, Black DM; FLEX Research Group: Efficacy of continued alendronate for fractures in women with and without prevalent vertebral fracture: the FLEX trial. J Bone Miner Res 2010, 25:976-982
13. Xue D, Li F, Chen G, Yan S, Pan Z: Do bisphosphonates affect bone healing? a meta-analysis of randomized controlled trials. J Orthop Surg Res 2014, 9:45

14. Kates SL, Ackert-Bicknell CL: How do bisphosphonates affect fracture healing? Injury 2016, 47 Suppl 1:S65-S68

15. Adler RA, El-Hajj Fuleihan G, Bauer DC, Camacho PM, Clarke BL, Clines GA, Compston JE, Drake MT, Edwards BJ, Favus MJ, Greenspan SL, McKinney R Jr, Pignolo RJ, Sellmeyer DE: Managing osteoporosis in patients on long-term bisphosphonate treatment: report of a Task Force of the American Society for Bone and Mineral Research. J Bone Miner Res 2016, 31:16-35

16. Diab DL, Watts NB: Bisphosphonate drug holiday: who, when and how long. Ther Adv Musculoskelet Dis 2013, 5:107-111

17. Ro C, Cooper O: Bisphosphonate drug holiday: choosing appropriate candidates. Curr Osteoporos Rep 2013, 11:45-51

18. Kawakami Y, Ii M, Matsumoto T, Kawamoto A, Kuroda R, Akimaru H, Mifune Y, Shoji T, Fukui T, Asahi M, Kurosaka M, Asahara T: A small interfering RNA targeting Lnk accelerates bone fracture healing with early neovascularization. Lab Invest 2013, 93: $1036-1053$

19. Fong K, Truong V, Foote CJ, Petrisor B, Williams D, Ristevski B, Sprague S, Bhandari M: Predictors of nonunion and reoperation in patients with fractures of the tibia: an observational study. BMC Musculoskelet Disord 2013, 14:103

20. Jing D, Hao X, Xu F, Liu J, Xu F, Luo E, Meng G: Effects of local delivery of BMP2, zoledronate and their combination on bone microarchitecture, biomechanics and bone turnover in osteoporotic rabbits. Sci Rep 2016, 6:28537

21. Kyllonen L, D'Este M, Alini M, Eglin D: Local drug delivery for enhancing fracture healing in osteoporotic bone. Acta Biomater 2015, $11: 412-434$

22. Brandi ML: Healing of the bone with anti-fracture drugs. Expert Opin Pharmacother 2013, 14:1441-1447

23. Aghaloo T, Cowan CM, Chou YF, Zhang X, Lee H, Miao S, Hong N, Kuroda S, Wu B, Ting K, Soo C: Nell-1-induced bone regeneration in calvarial defects. Am J Pathol 2006, 169:903-915

24. Aghaloo T, Jiang X, Soo C, Zhang Z, Zhang X, Hu J, Pan H, Hsu T, Wu B, Ting K, Zhang X: A study of the role of nell-1 gene modified goat bone marrow stromal cells in promoting new bone formation. Mol Ther 2007, 15:1872-1880

25. Ting K, Vastardis H, Mulliken JB, Soo C, Tieu A, Do H, Kwong E, Bertolami CN, Kawamoto H, Kuroda S, Longaker MT: Human NELL-1 expressed in unilateral coronal synostosis. J Bone Miner Res $1999,14: 80-89$

26. Zhang X, Ting K, Bessette CM, Culiat CT, Sung SJ, Lee H, Chen F, Shen J, Wang JJ, Kuroda S, Soo C: Nell-1, a key functional mediator of Runx2, partially rescues calvarial defects in Runx2(+/-) mice. J Bone Miner Res 2011, 26:777-791

27. Zhang X, Kuroda S, Carpenter D, Nishimura I, Soo C, Moats R, Iida K, Wisner E, Hu F-Y, Miao S, Beanes S, Dang C, Vastardis H, Longaker M, Tanizawa K, Kanayama N, Saito N, Ting K: Craniosynostosis in transgenic mice overexpressing Nell-1. J Clin Invest 2002, 110:861-870

28. Desai J, Shannon ME, Johnson MD, Ruff DW, Hughes LA, Kerley MK, Carpenter DA, Johnson DK, Rinchik EM, Culiat CT: Nell1-deficient mice have reduced expression of extracellular matrix proteins causing cranial and vertebral defects. Hum Mol Genet 2006, 15:1329-1341

29. Shen J, James AW, Chung J, Lee K, Zhang JB, Ho S, Lee KS, Kim TM, Niimi T, Kuroda S, Ting K, Soo C: NELL-1 promotes cell adhesion and differentiation via Integrinbeta1. J Cell Biochem 2012 113:3620-3628

30. James AW, Shen J, Zhang X, Asatrian G, Goyal R, Kwak JH, Jiang L, Bengs B, Culiat CT, Turner AS, Seim Iii HB, Wu BM, Lyons K, Adams JS, Ting K, Soo C: NELL-1 in the treatment of osteoporotic bone loss. Nat Commun 2015, 6:7362 
31. Shen J, James AW, Zhang X, Pang S, Zara JN, Asatrian G, Chiang M, Lee M, Khadarian K, Nguyen A, Lee KS, Siu RK, Tetradis S, Ting K, Soo C: Novel Wnt regulator NEL-like molecule-1 antagonizes adipogenesis and augments osteogenesis induced by bone morphogenetic protein 2. Am J Pathol 2016, 186: 419-434

32. Zhang X, Peault B, Chen W, Li W, Corselli M, James AW, Lee M, Siu RK, Shen P, Zheng Z, Shen J, Kwak J, Zara JN, Chen F, Zhang H, Yin Z, Wu B, Ting K, Soo C: The Nell-1 growth factor stimulates bone formation by purified human perivascular cells. Tissue Eng Part A 2011, 17:2497-2509

33. Lu SS, Zhang X, Soo C, Hsu T, Napoli A, Aghaloo T, Wu BM, Tsou P, Ting K, Wang JC: The osteoinductive properties of Nell-1 in a rat spinal fusion model. Spine J 2007, 7:50-60

34. Li W, Lee M, Whang J, Siu RK, Zhang X, Liu C, Wu BM, Wang JC, Ting K, Soo C: Delivery of lyophilized Nell-1 in a rat spinal fusion model. Tissue Eng Part A 2010, 16:2861-2870

35. James AW, Shen J, Tsuei R, Nguyen A, Khadarian K, Meyers CA, Pan HC, Li W, Kwak JH, Asatrian G, Culiat CT, Lee M, Ting K, Zhang X, Soo C: NELL-1 induces Sca-1+ mesenchymal progenitor cell expansion in models of bone maintenance and repair. JCI Insight 2017, 2:92573

36. Li W, Zara JN, Siu RK, Lee M, Aghaloo T, Zhang X, Wu BM, Gertzman AA, Ting K, Soo C: Nell-1 enhances bone regeneration in a rat critical-sized femoral segmental defect model. Plast Reconstr Surg 2011, 127:580-587

37. Zhang Y, Velasco O, Zhang X, Ting K, Soo C, Wu BM: Bioactivity and circulation time of PEGylated NELL-1 in mice and the potential for osteoporosis therapy. Biomaterials 2014, 35:6614-6621

38. Kwak JH, Zhang Y, Park J, Chen E, Shen J, Chawan C, Tanjaya J, Lee S, Zhang X, Wu BM, Ting K, Soo C: Pharmacokinetics and osteogenic potential of PEGylated NELL-1 in vivo after systemic administration. Biomaterials 2015, 57:73-83

39. Ning B, Zhao Y, Buza Iii JA, Li W, Wang W, Jia T: Surgically induced mouse models in the study of bone regeneration: current models and future directions (Review). Mol Med Rep 2017, 15: $1017-1023$

40. Kooistra BW, Dijkman BG, Busse JW, Sprague S, Schemitsch EH, Bhandari M: The radiographic union scale in tibial fractures: reliability and validity. J Orthop Trauma 2010, 24 Suppl 1:S81-S86

41. Tanjaya J, Zhang Y, Lee S, Shi J, Chen E, Ang P, Zhang X, Tetradis S, Ting K, Wu B, Soo C, Kwak JH: Efficacy of intraperitoneal administration of PEGylated NELL-1 for bone formation. Biores Open Access 2016, 5:159-170

42. Grauer AL, Roberts CA: Paleoepidemiology, healing, and possible treatment of trauma in the medieval cemetery population of St. Helenon-the-Walls, York, England. Am J Phys Anthropol 1996, 100: $531-544$

43. Owen JL, Stephens D, Wright JG: Reliability of radiographic measurement of fracture angulation in children with femoral shaft fractures. Can J Surg 2007, 50:115-118

44. Shefelbine SJ, Simon U, Claes L, Gold A, Gabet Y, Bab I, Muller R, Augat P: Prediction of fracture callus mechanical properties using micro-CT images and voxel-based finite element analysis. Bone 2005, 36:480-488

45. Chung CG, James AW, Asatrian G, Chang L, Nguyen A, Le K, Bayani G, Lee R, Stoker D, Zhang X, Ting K, Peault B, Soo C: Human perivascular stem cell-based bone graft substitute induces rat spinal fusion. Stem Cells Transl Med 2014, 3:1231-1241
46. Zara JN, Siu RK, Zhang X, Shen J, Ngo R, Lee M, Li W, Chiang M, Chung J, Kwak J, Wu BM, Ting K, Soo C: High doses of bone morphogenetic protein 2 induce structurally abnormal bone and inflammation in vivo. Tissue Eng Part A 2011, 17:1389-1399

47. James AW, LaChaud G, Shen J, Asatrian G, Nguyen V, Zhang X, Ting K, Soo C: A review of the clinical side effects of bone morphogenetic protein-2. Tissue Eng Part B Rev 2016, 22:284-297

48. Dimitriou R, Jones E, McGonagle D, Giannoudis PV: Bone regeneration: current concepts and future directions. BMC Med 2011, 9:66

49. Emara KM, Diab RA, Emara AK: Recent biological trends in management of fracture non-union. World J Orthop 2015, 6:623-628

50. Fayaz HC, Giannoudis PV, Vrahas MS, Smith RM, Moran C, Pape HC, Krettek C, Jupiter JB: The role of stem cells in fracture healing and nonunion. Int Orthop 2011, 35:1587-1597

51. Nauth A, Schemitsch EH: Stem cells for the repair and regeneration of bone. Indian J Orthop 2012, 46:19-21

52. Hoang-Kim A, Gelsomini L, Luciani D, Moroni A, Giannini S: Fracture healing and drug therapies in osteoporosis. Clin Cases Miner Bone Metab 2009, 6:136-143

53. Kang H, Park YC, Yang KH: Paget's disease: skeletal manifestations and effect of bisphosphonates. J Bone Metab 2017, 24:97-103

54. Meganck JA, Begun DL, McElderry JD, Swick A, Kozloff KM, Goldstein SA, Morris MD, Marini JC, Caird MS: Fracture healing with alendronate treatment in the $\mathrm{Brtl} / \mathrm{+}$ mouse model of osteogenesis imperfecta. Bone 2013, 56:204-212

55. Brandi ML: Drugs for bone healing. Expert Opin Investig Drugs 2012, 21:1169-1176

56. Solomon DH, Hochberg MC, Mogun H, Schneeweiss S: The relation between bisphosphonate use and non-union of fractures of the humerus in older adults. Osteoporos Int 2009, 20:895-901

57. Harris JM, Chess RB: Effect of pegylation on pharmaceuticals. Nat Rev Drug Discov 2003, 2:214-221

58. Horner EA, Kirkham J, Wood D, Curran S, Smith M, Thomson B, Yang XB: Long bone defect models for tissue engineering applications: criteria for choice. Tissue Eng Part B Rev 2010, 16:263-271

59. McKibbin B: The biology of fracture healing in long bones. J Bone Joint Surg Br 1978, 60-B:150-162

60. Bao Q, Chen S, Qin H, Feng J, Liu H, Liu D, Li A, Shen Y, Zhao Y, Li J, Zong Z: An appropriate Wnt/beta-catenin expression level during the remodeling phase is required for improved bone fracture healing in mice. Sci Rep 2017, 7:2695

61. Buckwalter JA, Hunziker EB: Orthopaedics: healing of bones, cartilages, tendons, and ligaments: a new era. Lancet 1996, 348 Suppl 2: sII18

62. Pang S, Shen J, Liu Y, Chen F, Zheng Z, James AW, Hsu CY, Zhang H, Lee KS, Wang C, Li C, Chen X, Jia H, Zhang X, Soo C, Ting K: Proliferation and osteogenic differentiation of mesenchymal stem cells induced by a short isoform of NELL-1. Stem Cells 2015, 33:904-915

63. Oetgen ME, Merrell GA, Troiano NW, Horowitz MC, Kacena MA: Development of a femoral non-union model in the mouse. Injury 2008, 39:1119-1126

64. Yin G, Sheu TJ, Menon P, Pang J, Ho HC, Shi S, Xie C, Smolock E, Yan C, Zuscik MJ, Berk BC: Impaired angiogenesis during fracture healing in GPCR kinase 2 interacting protein-1 (GIT1) knock out mice. PLoS One 2014, 9:e89127

65. Johnell O, Kanis JA: An estimate of the worldwide prevalence and disability associated with osteoporotic fractures. Osteoporos Int 2006, 17:1726-1733 